\title{
Study on Jamin Effect in the Low Permeability Reservoir
}

\section{Y.J.TIAN \& C.X.OUYANG*}

A. A. Key Laboratory of Oil and Gas Drilling Projects in Hubei province, Yangtze University, Wuhan, Hubei, China

Institute of Petroleum Engineering, Yangtze University, Wuhan, Hubei, China

X.S.CAI

A. A. Key Laboratory of Reservoir Protection and Reconstruction, Yangtze University, Wuhan, Hubei, China

ABSTRACT: It is vulnerable to be damaged by Jamin effect in the process of flood development, so it is difficult to extract oil in the low permeability and the recovery efficiency is low. Taking a well field in Tarim Oilfield as an example, Jamin effect evaluation experiment and evaluation standard are designed and set by water/oil displacement experiment of natural standstone. Using Jamin index to estimate Jamin damage, sifting kinds of surfactants, carry out laboratory test of redcuing Jamin effect. The experimental results show that the research well field has middle-strong Jamin effect, which can be reduced effectively by reducing the interfacial tension. The results show that the method of reducing oil-water interfacial tension by adding $0.01 \%$ Gemini Surfactants to injected water can graeatly reduce Jamin effect and improve the recovery efficiency.

KEYWORD: Jamin effect; low permeability; water/oil displacement; surfactants

\section{INTRODUCTION}

When immiscible two-phase like liquid-liquid phase or gas-liquid phase seepaging in rock pore, phase interface moving through the capillary pore narrow throat need to overcome the capillary resistance, and the resistance effect called Jamin effect. The strength of the development of Jamin effect degree is controlled by the size of rocks throat in reservoir. Because of its small throat of low permeability sandstone reservoir, the probability of Jamin effect is large in waterflood development [1]. The lower reservoir permeability, the more outstanding Jamin effect is. The research wellblock reservoir in Tarim oilfield is a typical low permeability sandstone reservoir, which belongs to the tiny pore throat type, strong heterogeneity, strong Jamin effect in water flooding development reservoir.

Therefore, in order to discusses the Jamin effect of low permeability sandstone oilfield development, the low permeable sandstone cores taken from the Tarim basin has carried on the microscopic model of water/oil displacement experiment. Reservoir sandstone clastic constituents in the study area are mainly quartz, feldspar and cuttings. The rock composition maturity is low. The rock types are mainly lithic sandstone, while few samples are the lithic quartz sandstone.

\section{MECHANISM OF JAMIN EFFECT}

In rock pore percolation process of immiscible twophase flow, when the liquid bead (or bubble) moving to the narrow mouth of reservoir pore, due to its diameter greater than the diameter of the shouted, it deforms. It leads to the liquid drop (or bubble) at both ends of the curved liquid surface have different curvature, then, additional capillary resistance will be produced (Figure 1):

$$
\mathrm{P}=2 \sigma\left(1 / \mathrm{R}^{\prime \prime}-1 / \mathrm{R}^{\prime}\right) \text {. }
$$

We call this kind of additional resistance "fluid resistance effect" or "Jamin effect". Only when both ends of the curvature become inconsistent, liquid drop (or bubble) can get through the tiny roared.

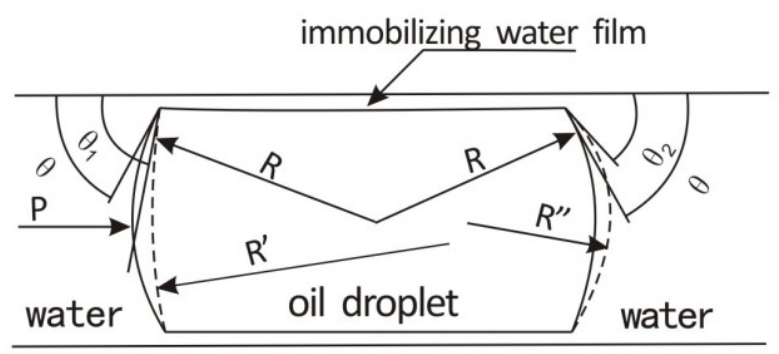

A 


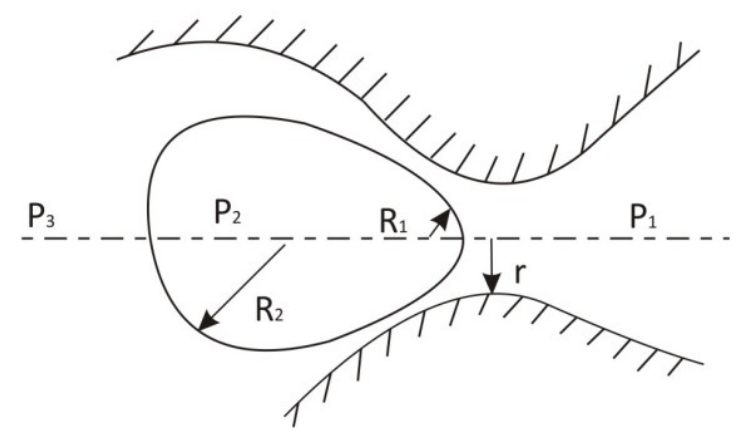

B

Figure 1, Extra resistance of oil drop(A) and Jamin effect(B) in capillary

Where: $\sigma=$ The oil-water interfacial tension;

$\mathrm{R}=$ Resting spherical radius $(\mathrm{m})$;

$\mathrm{R}^{\prime}=$ The back-end spherical radius ( $\left.\mathrm{m}\right)$;

$\mathrm{R}^{\prime \prime}=$ The front-end spherical radius $(\mathrm{m})$;

$\mathrm{P}=$ The oil displacement differential pressure $(\mathrm{MPa})$;

$\theta=$ The static contact Angle $\left(^{\circ}\right)$;

$\theta_{1}=$ The back-end dynamic contact Angle $\left(^{\circ}\right)$;

$\theta_{2}=$ The front dynamic contact Angle $\left(^{\circ}\right)$;

$\mathrm{P}_{1}=$ Ball steep front end to wall spherical capillary

force $(\mathrm{MPa})$;

$\mathrm{P}_{2}=$ Cylindrical surface point to tube capillary force $(\mathrm{MPa})$

$\mathrm{P}_{3}=$ Pearl bubble backend to wall spherical capillary

force $(\mathrm{MPa})$;

$\mathrm{R}_{1}=$ Pearl bubble back-end meniscus radius (m);

$\mathrm{R}_{1}=$ Pearl bubble front meniscus radius (m);

$r=$ Throat radius $(\mathrm{m})$.

\section{AFFECTING FACTORS OF JAMIN EFFECT}

Many factors affect the Jamin effect, and the relationship is complex and varied, this paper mainly discusses the following factors.

(1) Poroperm characteristics. The relationship between Jamin effect and petrophysical is very close. The lower permeability, the more serious reservoir heterogeneity and Jamin effect become. Formation permeability is associated with the heterogeneity. The smaller heterogeneity, the greater permeability is. At the same time, the size of the formation porosity, to a certain extent, also determines the size of the formation permeability [2]. On the contrary, the strong heterogeneity and maldistributed particles are most likely to form the pore structure of larger pore throat ratio. The probability of Jamin effect is greater.

(2) Pore throat ratio, porosity and cross-section area. Permeability and porosity are proportional to cross-section area, and are inversely proportional to the pore throat ratio. Under the circumstance that the pore throat ratio is constant, the greater the porosity and the cross-sectional area, the greater the permeability is; under the circumstance that formation porosity and the cross-sectional area are constant, the greater the pore throat ratio, the smaller the permeability is [3]. Jamin effect is more outstanding.

(3) Liquidus invasion depth and water saturation. The deeper the invasion depth, the higher the water saturation is. The stronger the fluid resistance damage, the more severe Jamin effect is [4].

(4) Interfacial tension. Adding surfactant can not only reduce the oil-water interfacial tension, but als change the wettability. The capillary resistance becomes a driving force, so as to effectively reduce the fluid resistance index.

\section{CORRESPONDING MEASURE OF JAMIN EFFECT}

Jamin effect increases with permeability increasing, and increases with oil saturation decreasing when the dispersed oil droplets/beads increase. That is to say, in the development of low permeability and extralow permeability oil field, Jamin effect increase with development degree deepening. It is difficult to eliminate Jamin effect under the condition of current technology. Therefore, as far as possible to prevent or weaken the Jamin effect is an effective way to efficient development of low permeability oilfield.

In the actual production process, we can ease the Jamin phenomenon by increasing the injection pressure. But when the liquid drop/ bubble move to smaller throat, this phenomenon will appear again. In the actual production, we cannot unlimited increase injection pressure. The calculation formula for the Jamin effect:

$$
\mathrm{P}=2 \sigma\left(1 / \mathrm{R}^{\prime \prime}-1 / \mathrm{R}^{\prime}\right) \text {. }
$$

So by the formula, from the other point of view can be seen, there are two ways to mitigate the damage. One is to reduce the oil-water interfacial tension, another is to expand the pore radius. In the scene, there are many methods to expand the radius during the actual production, such as acidification, etc. Under laboratory conditions, we mainly aimed at reducing the oil-water interfacial tension experiment.

\subsection{Screening of surfactants}

Through interfacial tension determination of sodium dodecyl benzene sulfonate, mahogany sulfonate, petroleum carboxylate, gemini surfactant etc, selected the most suitable surfactant [5]. By means of measuring interfacial tension, the surfactant can be optimzed. The instrumentation is TEXAS500 Spinning Drop Interface tensiometer. 
Table 1, Oil-water interfacial tension measurement results of surfactants

\begin{tabular}{|c|c|c|}
\hline The surfactant & $\begin{array}{c}\text { Concentration } \\
(\%)\end{array}$ & $\begin{array}{c}\text { Interfacial } \\
\text { tension }(\mathrm{mN} / \mathrm{m})\end{array}$ \\
\hline $\begin{array}{c}\text { Sodium dodecyl } \\
\text { benzene sulfonate }\end{array}$ & 0.10 & 0.48 \\
\hline $\begin{array}{c}\text { Mahogany } \\
\text { sulfonate }\end{array}$ & 0.05 & $9.53 \times 10^{-2}$ \\
\hline $\begin{array}{c}\text { Petroleum } \\
\text { carboxylate }\end{array}$ & 0.05 & $1.72 \times 10^{-2}$ \\
\hline $\begin{array}{c}\text { Gemini } \\
\text { surfactant }\end{array}$ & 0.02 & $1.96 \times 10^{-2}$ \\
\hline $\begin{array}{c}\text { Gemini } \\
\text { surfactant }\end{array}$ & 0.01 & $3.687 \times 10^{-3}$ \\
\hline
\end{tabular}

From table 1, different types, different concentrations of surfactants interfacial tension are different. Among them, interfacial tension of $0.01 \%$ Gemini surfactant is minimum. Therefore choose it as a protectant, and conduct core water/oil displacement experiment to verify the effect of the surfactant.

\subsection{The surfactant to improve Jamin effect experiment}

Experimental process as follows:

(1) Saturate the evacuated core with simulated formation water more than three hours;

(2) Displace the core with light weight aviation kerosene which has been filtrated by filter paper until no water discharge. Collect and measure the volume of discharged water to calculate irreducible water saturation $\mathrm{S}_{\mathrm{wi}}$;

(3) Record time, velocity, pressure peak value $\left(\Delta \mathrm{P}_{\text {odw }}\right)$ and stable pressure difference value during the displacement;

(4) Clean kerosene in core and bake out the core at about 80 degrees Celsius for hours. Saturate the evacuated core with filtrated lightweight aviation kerosene more than three hours. Displace the core with simulated formation water;

(5) Record time, velocity, pressure peak value $\left(\Delta \mathrm{P}_{\mathrm{wdo}}\right)$ and stable pressure difference value during the displacement;

(6) Calculate the before injected surfactant of the Jamin index: $\mathrm{I}_{\mathrm{J}}=\Delta \mathrm{P}_{\mathrm{w}} / \Delta \mathrm{P}_{\mathrm{o}}$;

(7) Clean core again and bake out the core at about 80 degrees Celsius for hours. Saturate the evacuated core with filtrated lightweight aviation kerosene more than three hours. Displace the evacuated core with simulated formation water with $0.01 \%$ Gemini surfactant for two pore volume, and shut in for 1 hour;

(8) Open the pump, and displace the core with simulated formation water with $0.01 \%$ Gemini surfactant again. Record time, velocity, pressure peak value $\left(\Delta \mathrm{P}_{\mathrm{wdo}}{ }^{\prime}\right)$ and stable pressure difference value during the displacement;
(9) Calculate the after injected surfactant of the Jamin index: $\mathrm{I}_{\mathrm{J}}{ }^{\prime}=\Delta \mathrm{P}_{\mathrm{w}}{ }^{\prime} / \Delta \mathrm{P}_{\mathrm{o}}{ }^{\prime}$;

(10) Draw tables and figures of Jamin damage evaluation to compare of before and after injected surfactant Jamin index.

Jamin effect evaluation standard[6] are shown in table 2 .

Table 2, Evaluation standard of Jamin effect

\begin{tabular}{|c|c|c|c|c|}
\hline $\begin{array}{c}\text { Jamin effect } \\
\text { extent }\end{array}$ & No & Weak & Medium & Strong \\
\hline $\begin{array}{c}\text { Jamin Index } \\
\mathrm{I}_{\mathrm{T}}\end{array}$ & $<1.0$ & $1.0-1.5$ & $1.5-2.0$ & $>2.0$ \\
\hline
\end{tabular}

Sample experimental results are shown in Table3.

Table 3, Before / after injected surfactant Jamin damage evaluation results comparison table

\begin{tabular}{|c|c|c|c|}
\hline Sample number & XX-1 & XX-2 & XX-3 \\
\hline Porosity $(\%)$ & 13.503 & 11.262 & 13.865 \\
\hline $\begin{array}{c}\text { Gas permeability } \\
\left(10^{-3} \mu \mathrm{m}^{2}\right)\end{array}$ & 7.610 & 1.824 & 168.923 \\
\hline $\begin{array}{c}\text { Initial permeability } \\
\left(10^{-3} \mu \mathrm{m}^{2}\right)\end{array}$ & 0.334 & 0.140 & 22.031 \\
\hline $\mathrm{I}_{\mathrm{J}}$ & $1.75-2.42$ & $2.22-3.60$ & $1.51-2.31$ \\
\hline $\begin{array}{c}\text { Jamin effect extent } \\
(\text { before })\end{array}$ & medium-strong & strong & medium-strong \\
\hline $\mathrm{I}_{\mathrm{J}}{ }^{\prime}$ & $1.33-1.87$ & $2.11-2.73$ & $0.88-1.24$ \\
\hline $\begin{array}{c}\text { Jamin effect extent } \\
(\text { after })\end{array}$ & weak-medium & strong & no-weak \\
\hline
\end{tabular}
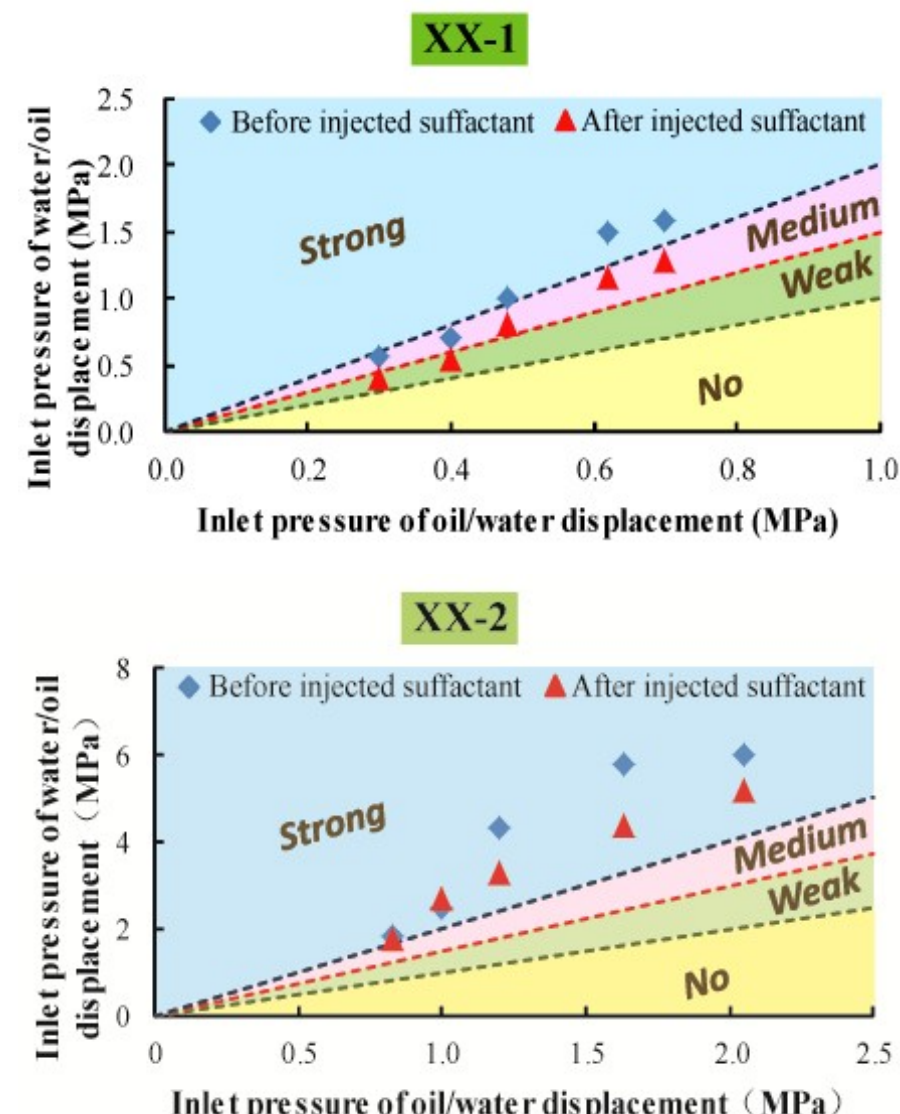

Inle t pressure of oil/water dis placement (MPa) 


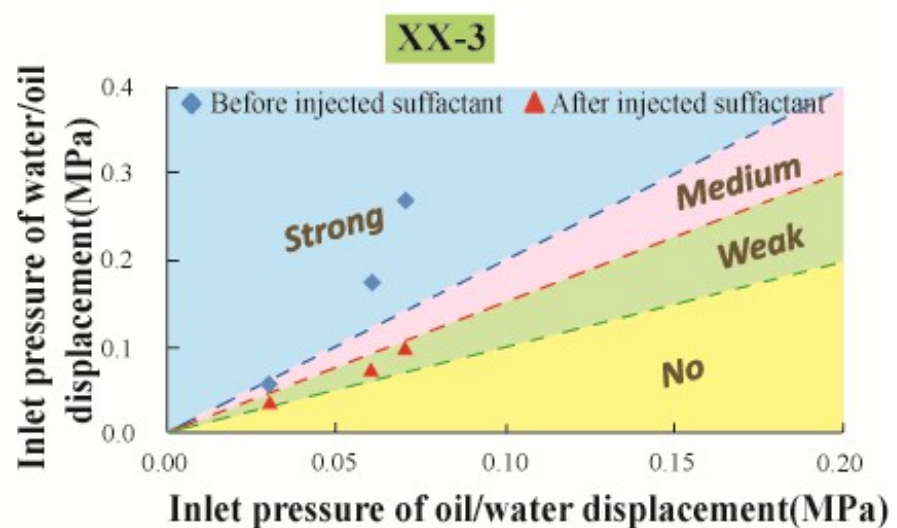

Figure 2, Before/ after injected surfactant Jamin damage evaluation results of core sample in study area

From Figure2, after injecting protective agent, the extent of Jamin damage is reduced to a certain extent. It shows that adding $0.01 \%$ Gemini surfactant can effectively reduce the oil-water interfacial tension, thereby effectively reducing Jamin damage. But Jamin damage reduction degree of XX-1 and $\mathrm{XX}-2$ core samples is low and the effect is not obvious. Jamin damage of XX-3 rock samples is obviously slow down. For this phenomenon, the author thinks that in the case of smaller pore radius, protective agent to improve the interfacial tension effects do not strong enough to significantly reduce the droplet through roared resistance. When the pore radius is larger, reducing the oil-water interfacial tension has great influence to liquid bead deformation go through the throat. So, the impact on the Jamin effect should be based on prevention, especially to the poor storage and permeability reservoir. It should pay special attention to leak proof well mud loss work, avoiding excessive mud filtrate into the formation to cause difficult to mitigation Jamin damage. For the reservoir damage had been done, under good conditions of the reservoir permeability, it can try to inject the protective agent.

\section{CONCLUSIONS}

(1) The strength of the development of Jamin effect degree is controlled by the size of rocks throat in reservoir. Because of its small throat of low permeability sandstone reservoir, the probability of
Jamin effect is large in waterflood development. The lower reservoir permeability, the more outstanding Jamin effect is.

(2) Affecting Factors of Jamin Effect are Poroperm characteristics, pore throat ratio, porosity, interface agent, liquid invasion depth and water saturation, interfacial tension etc.

(3) Reducing interfacial tension between oil and water is an effective way to slow down Jamin effect. That is to say, under laboratory conditions, injecting water with $0.01 \%$ preferred Gemini surfactant to reduce the oil-water interfacial tension can significantly weaken the Jamin effect. Reduce injection pressure, reduce the mining cost, and improve the economic benefits of oil reservoir.

(3) Jamin effect should be based on prevention, especially to the poor storage and permeability reservoir. It should pay special attention to leak proof well mud loss work. For the reservoir damage had been done, under good conditions of the reservoir permeability, it can try to inject the protective agent.

\section{REFERENCES}

[1] ZHANG Zhen-hua, YAN Jie-nian. The study of influence factors and predicting method of water blocking in the lowpermeable sandstone formations. Petroleum Exploration and Development, 2000, 27(3): 76-78.

[2] WANG Rui-fei, CHEN Jun-bin, SUN Wei. Jamin Effect in the Ultra -low Permeability Sandstone Oil Field Development: An Example from Yanchang Formation of Mesozoic Strata. Geological Science and Technology Information, 2008, 27(5): 82-86.

[3] Hassan Bahrami, Raza Rezaee M, Delair Nazhat, et al. Effect of water blocking damage on flow efficiency and productivity in tight gas reservoirs.SPE 142283, 2011.

[4] [4] FAN Xiang-yu, XIA Hong-quan, CHEN Ping, et al. Study on calculating method of invasion depth of mud solid phase, Natural Gas Industry, 2006, 26(3): 75-77.

[5] CHE Hong-chang, REN Yao-yu, LIU Han-ping, et al. Laboratory study on oil displacement with active water in Long hu pao Oil field, Lithologic Reservoirs, 2011,23(2):128-131.

[6] LV Lu, OUYANG Chuan-xiang, ZHANG Zhi-jun et al. Jamin Effect Evaluation Experiment and Release Measures of Low Permeability Sandstone Reservoir: An Example from Baxtopu Donghetang Formation within Tarim Oilfield. Geological Science and Technology Information, 2012, 31(2): 80-83 